\title{
Abundances and Gradients in M31 \\ - A Chemical Study of Planetary Nebulae in the Substructures
}

\section{Xuan Fang ${ }^{1,2}$, Rubén García-Benito ${ }^{3}$, Martín A. Guerrero ${ }^{3}$, Xiaowei Liu ${ }^{4,5}$, Yong Zhang ${ }^{1,6}$ and Haibo Yuan}

${ }^{1}$ Laboratory for Space Research, University of Hong Kong, Pokfulam Road, Hong Kong, China email: fangx@hku.hk

${ }^{2}$ Department of Earth Sciences, University of Hong Kong, Pokfulam Road, Hong Kong, China

${ }^{3}$ Instituto de Astrofísica de Andalucía, Glorieta de la Astronomía s/n, 18008, Granada, Spain

${ }^{4}$ Department of Astronomy, School of Physics, Peking University, Beijing 100871, China

${ }^{5}$ Kavli Institute for Astronomy and Astrophysics, Peking University, Beijing 100871, China

${ }^{6}$ Department of Physics, University of Hong Kong, Pokfulam Road, Hong Kong, China

${ }^{7}$ Department of Astronomy, Beijing Normal University, Beijing 100875, China

\begin{abstract}
We present deep optical spectroscopy of seven planetary nebulae (PNe) in the substructures of M31, three in the Northern Spur and four associated with the Giant Stream. The spectra were obtained with the OSIRIS spectrograph on the $10.4 \mathrm{~m}$ GTC. The detection of the [O III] $\lambda 4363$ auroral line in all PNe of our sample enables reliable abundance determinations. Our targets have low $\mathrm{N} / \mathrm{O}(<0.5)$ and $\mathrm{He} / \mathrm{H}$ ratios, indicating that they are probably Type II $\mathrm{PNe}$. The PNe in our sample have rather homogeneous oxygen abundances, with an average value of $8.56 \pm 0.10$. Based on the abundances as well as the spatial and kinematical information of our targets, we speculate that the Northern Spur and the Giant Stream might have the same origin. We raise a hypothesis that the dwarf satellite M32 might be responsible for these two substructures. New observations have recently been made to assess this hypothesis.
\end{abstract}

Keywords. galaxies: abundances, galaxies: evolution, galaxies: individual (M31), ISM: abundances, planetary nebulae: general, stars: evolution

\section{Introduction}

Planetary nebulae (PNe) are excellent tools to study the chemistry, kinematics, and stellar populations of host galaxies. Being bright in emission lines (e.g., [O III] $\lambda 5007$ ), PNe are easily detected in the Andromeda Galaxy (M31), the nearest (780 kpc) large spiral system. Abundances of PNe in the disk and bulge of M31 have been studied for decades (e.g., Jacoby \& Ford 1986; Jacoby \& Ciardullo 1999; Kwitter et al. 2012) and abundance gradients were derived, which help to understand chemical evolution of M31. Recent observations using the $10 \mathrm{~m}$ class telescope have pushed abundance determination of M31 PNe to higher levels of accuracy and invoked novel ideas of formation and evolution of M31 (Balick et al. 2013; Corradi et al. 2015; Fang et al. 2015).

Although chemical abundances of disk PNe in M31 have been extensively studied, those associated with the substructures was largely ignored. It has been widely accepted that large galaxies evolve from merging of smaller galaxies (White 1978). The relics of galaxy interaction can be seen in the outskirts of galaxies in the form of various substructures such as tidal tails or stellar streams. Numerous substructures have been discovered in the outskirts of M31 (e.g., Ibata et al. 2001; McConnachie et al. 2009). The exact origin 


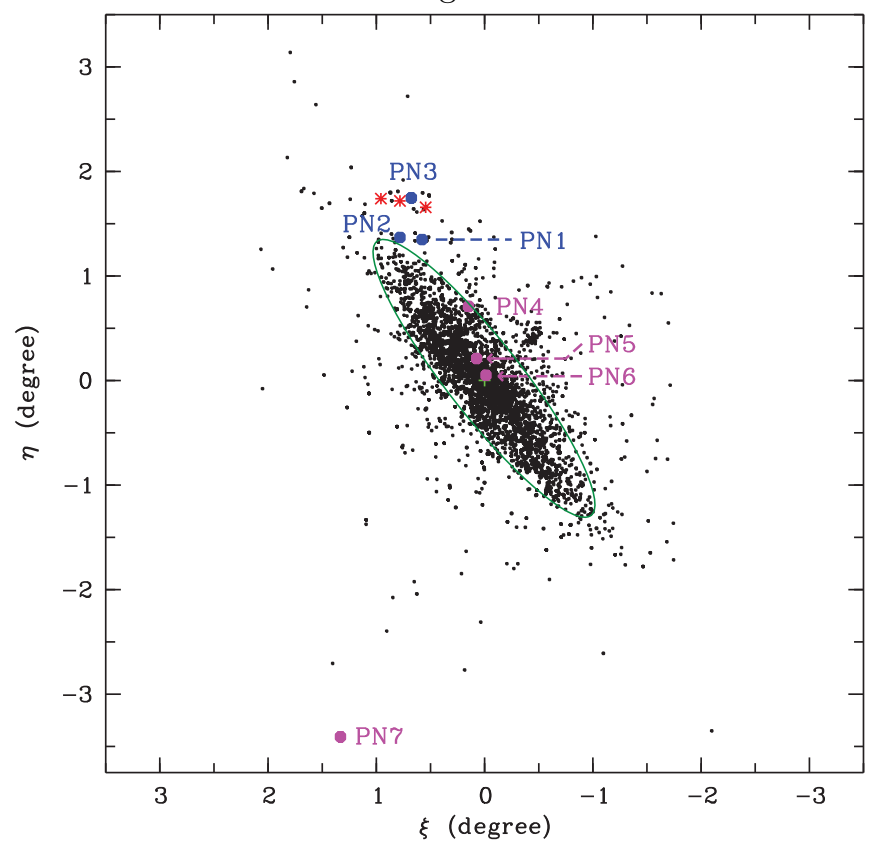

Figure 1. Distribution of PNe in M31. Objects are mostly from the catalogs of Merrett et al. (2006) and Yuan et al. (2010). Red asterisks are the three Northern Spur PNe observed by Fang et al. (2013). The PNe observed by Fang et al. (2015), three in the Northern Spur (blue filled circles, named PN1-3) and four associated with the Giant Stream (magenta fill circles, named PN4-7) are highlighted. The green ellipse is a $2^{\circ}$-radius $(27.4 \mathrm{kpc})$ round disk centered on M31 with an inclination angle of $77.7^{\circ}$ and a position angle of $37.7^{\circ}$.

of the southern stellar stream (also known as the Giant Stream) and the Northern Spur, the two most prominent stellar substructures of M31, are still unclear, although possible connection of these two has been proposed (Merrett et al. 2003). Detailed studies of PNe in the substructures may provide important clues to the assemblage history of the galaxy.

We have tried to obtain deep optical spectra of PNe in the substructures of M31. Our first effort was made with the $5.1 \mathrm{~m}$ Hale Telescope to observe three PNe in the Northern Spur, but with limited data quality (Fang et al. 2013). The follow-up observations were carried out using the $10.4 \mathrm{~m}$ Gran Telescopio Canarias at Observatorio del Roque de los Muchachos (ORM, La Palma). The observations and results are presented in the following sections.

\section{Observations}

Target Selection. Our targets were selected according to kinematics (see Figure 7 in Fang et al. 2015): six were selected from the catalog of Merrett et al. (2006), and the other one was identified by the Large Sky Area Multi-Object Fiber Spectroscopic Telescope (LAMOST; Yuan et al. 2010). Three are in the Northern Spur and the other four are kinematically associated with the Giant Stream (see Figure 1). The sky-projected galactocentric distances range from 0.7 to $50.2 \mathrm{kpc}$, and magnitudes $m(\lambda 5007) \sim 20.48-21.51$. Observations and Data Reduction. Observations of the seven PNe were made with the Optical System for Imaging and low-intermediate-Resolution Integrated Spectroscopy (OSIRIS) at the $10.4 \mathrm{~m}$ Gran Telescopio Canarias (GTC), from 2014 August 23 to 29 (2014B) in service mode. The seeing is $0.5-1.0$ arcsec during the observations. The grism $\mathrm{R} 1000 \mathrm{~B}$ and a 1 arcsec-wide long slit were used. The detector of OSIRIS consists of a mosaic of two Marconi CCDs with $2048 \times 4096$ pixels each. The pixel size is $15 \mu \mathrm{m}$ 

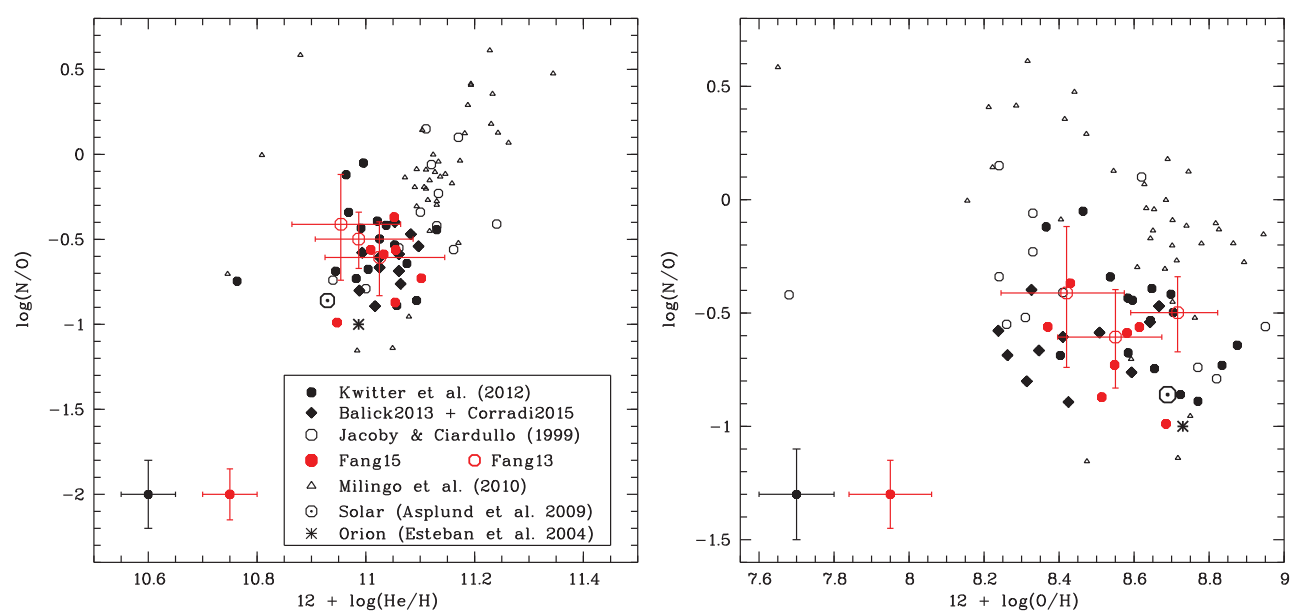

Figure 2. Left: $\log (\mathrm{N} / \mathrm{O})$ vs. $12+\log (\mathrm{He} / \mathrm{H})$. Right: $\log (\mathrm{N} / \mathrm{O})$ vs. $12+\log (\mathrm{O} / \mathrm{H})$. Different symbols represent different PN samples (see legend). Explanation of data sets is given in the text. Red open circles (along with error bars) are the three Northern Spur PNe observed by Fang et al. (2013, Fang13), and red filled symbols are the seven PNe observed by Fang et al. (2015, Fang15). Representative error bars of our targets and those of Kwitter et al. (2012, also Balick et al. 2013 and Corradi et al. 2015) are given in the lower-left corner.

$(\sim 0.127$ arcsec $)$. CCD data were $2 \times 2$ binned in the standard observing mode. This instrument setup gives a wavelength coverage $3630-7800 \AA$ with a spectral resolution of FWHM $6 \AA$ at $2.06 \AA$ pixel $^{-1}$. For each PN, four 20-40 min exposures were made. Exposures of the spectrophotometric standard stars were also made for each target. Arc lines of neon and $\mathrm{HgAr}$ lamps were obtained for wavelength calibration. All data were then reduced following standard procedures using IRAF (see details in Fang et al. 2015). The 1D spectrum of each target $\mathrm{PN}$ was finally extracted for analysis.

\section{Results}

Abundance Correlations. Elemental abundances of $\mathrm{He}, \mathrm{O}, \mathrm{N}, \mathrm{Ne}, \mathrm{S}$ and Ar relative to hydrogen were derived for our target PNe. Figure 2 show the N/O versus $\mathrm{He} / \mathrm{H}$ and $\mathrm{O} / \mathrm{H}$. Our sample are compared with the disk PNe observed by Jacoby \& Ciardullo (1999), Kwitter et al. (2012), Balick et al. (2013) and Corradi et al. (2015). The Galactic Type I PNe of Milingo et al. (2010) are also presented for purpose of comparison. Our sample have low $\mathrm{N} / \mathrm{O}(<0.5)$ and $\mathrm{He} / \mathrm{H}$ ratios, indicating they are probably Type II (i.e., their progenitor stars may have low masses). Not only our substructure PNe, but also those recently observed M31 disk PNe seem to be Type II.

Abundance Gradient. The oxygen gradient of M31 is presented in Figure 3. Our substructure PNe generally have consistent oxygen abundances within the errors, and have an average value of $8.56 \pm 0.12$. The oxygen abundance of target PN7, whose projectioncorrected galactocentric distance might be $\geqslant 100 \mathrm{kpc}$, is 0.11 dex lower than the solar value (8.69, Asplund et al. 2009). Abundances of PN5 and PN6 are higher than the disk and bulge samples of Jacoby \& Ciardullo (1999) and Sanders et al. (2012) at similar distances. The homogeneous distribution of oxygen in our sample indicates that the Northern Spur and the Giant Stream may have the same origin. We speculate that these two substructures might have been tidally stripped from the dwarf satellite M32, given its spatial position. For the disk PNe, oxygen gradient has a negative value $\left(-0.011 \pm 0.004 \mathrm{dex} \mathrm{kpc}^{-1}\right.$; Kwitter et al. 2012) within $50 \mathrm{kpc}$, but tends to be flattened further out. Although PN6 


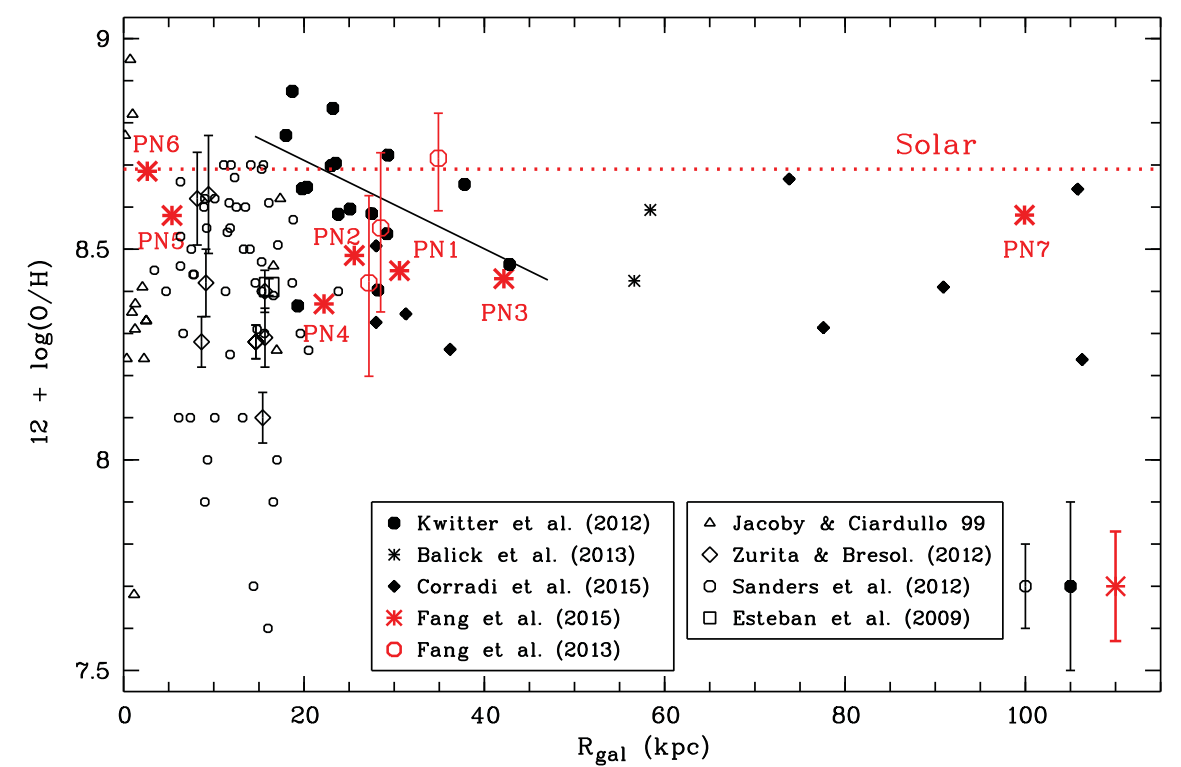

Figure 3. Oxygen abundance gradient of $\mathrm{PNe}$ and $\mathrm{H}$ II regions in M31. Our targets are red symbols, and their ID PN1-7 are the same as in Figure 1. Open diamonds are H II regions observed by Zurita \& Bresolin (2012), and open square is the H II region K932 studied by Esteban et al. (2009). The other symbols are PNe. Explanation of the other data sets is given in the text. The black straight line is a linear fit to the disk sample of Kwitter et al. (2012). The horizontal red dotted line is the solar value of Asplund et al. (2009).

seems to have higher $\mathrm{O} / \mathrm{H}$ than the other targets in our sample, its abundance uncertainty could be relatively larger due to its proximity to the galaxy center.

\section{New Observations}

We have obtained new GTC optical spectra of another 11 M31 PNe in 2016B: three in the Northern Spur, five associated with the Giant Stream, two in the dwarf satellite M32, and one in the outer halo. We have now 18 substructure PNe observed with the GTC. A systematic study of nebular abundances and properties of progenitor stars using photoionization modeling is underway (X. Fang et al., in preparation). We well also assess whether M32 is responsible for the two substructures.

\section{References}

Asplund, M., Grevesse, N., Sauval, A.-J., \& Scott, P. 2009, ARA\&A, 47, 481

Balick, B., Kwitter, K. B., Corradi, R. L. M., \& Henry, R. B. C. 2013, ApJ, 774, 3

Corradi, R. L. M., Kwitter, K. B., Balick, B., et al. 2015, ApJ, 807, 181

Esteban, C., Bresolin, F., Peimbert, M., et al. 2009, ApJ, 700, 654

Fang, X., García-Benito, R., Guerrero, M. A., et al. 2015, ApJ, 915, 69

Fang, X., Zhang, Y., García-Benito, R., Liu, X.-W., \& Yuan, H.-B. 2013, ApJ, 774, 138

Ibata, R. A., Irwin, M. J., Lewis, G. F., et al. 2001, Natur, 412, 49

Jacoby, G. H. \& Ciardullo, R. 1999, ApJ, 515, 169

Jacoby, G. H. \& Ford, H. C. 1986, ApJ, 304, 490

Kwitter, K. B., Lehman, E. M. M., Balick, B., \& Henry, R. B. C. 2012 ApJ, 753, 12

McConnachie, A. W., Irwin, M. J., Ibata, R. A., et al. 2009, Natur, 461, 66

Merrett, H. R., Kuijken, K., Merrifield, M. R., et al. 2003, MNRAS, 346, L62

Merrett, H. R., Merrifield, M. R., Douglas, N. G., et al. 2006, MNRAS, 369, 120

Milingo, J. B., Kwitter, K. B., Henry, R. B. C., \& Souza, S. P. 2010, ApJ, 711, 619

Sanders, N. E., Caldwell, N., McDowell, J., \& Harding, P. 2012, ApJ, 758, 133 
White, S. D. M. 1978, MNRAS, 184, 185

Yuan, H.-B., Liu, X.-W., Huo, Z.-Y., et al. 2010, RAA, 10, 599

Zurita, A. \& Bresolin, F. 2012, MNRAS, 427, 1463

\section{Discussion}

ARNABOLDI: As PI of the PN.S, I congratulate for the project to follow up PNs from Merrett's survey. Do you have plans to follow up discovery of PNs in the halo of M31?

LIU: LAMOST has hitherto surveyed a few thousand M31/M33 PNe and candidates, and obtained positive detections for over a thousand of them, including some new identifications (Yuan et al. 2010, RAA, 10, 599; Xiang et al., this volume). Some of the GTC targets reported in this talk were selected from the LAMOST new findings.

StAnghellini: You found that your targets do not have enhanced $\mathrm{He} / \mathrm{H}$ nor N/O, and you defined them as Type II. Could they be Type III, belonging to a thick disk/high velocity sample?

STERling: Two questions: How many magnitudes below the bright PNLF cutoff are the PNe in your sample? Secondly, you detected C II 4267 in some targets. Did you also detect O II ORLs so that you could determine $\mathrm{C} / \mathrm{O}$ ratios in some objects in your sample?

FANG: Most PNe in our sample are below the PNLF cutoff; 2) We have detected some weak features whose wavelengths agree with the O II ORLs, but low resolution $(\mathrm{R} \sim$ 1000) of the spectra prevents a further study of the C/O ORL ratios. 